\title{
Differential diagnostic CYFRA 21-1 level for benign and malignant pleural effusions: a meta-analysis in the Chinese population
}

Rong Biaoxue ${ }^{1}$, Yang Shuanying, Cai Xiguang ${ }^{2}$, Zhang Wei ${ }^{3}$, Li Wei ${ }^{1}$

\author{
1Department of Respiratory Medicine, The Second Affiliated Hospital of Xi'an Jiaotong \\ University, Xi'an, China \\ 2Department of Respiratory Medicine, The People's Hospital of Gansu Province, Lanzhou, \\ Gansu, China \\ 32Department of Thoracic Surgery, The Second Affiliated Hospital of Xi'an Jiaotong \\ University, Xi'an, China
}

Submitted: 9 April 2011

Accepted: 5 December 2011

Arch Med Sci 2012; 8, 5: 756-766

DOI: 10.5114 /aoms.2012.30831

Copyright (C) 2012 Termedia \& Banach

\section{Abstract}

Introduction: Many studies have investigated the usefulness of cytokeratin 19 fragments (CYFRA 21-1) in pleural fluid for the differential diagnosis of benign (BPE) and malignant pleural (MPE) effusions. In the present meta-analysis, the reported studies on the diagnosis between CYFRA 21-1 and pleural effusion were assessed to summarize the diagnostic characteristics of CYFRA 21-1 in Chinese patients. Material and methods: The data sources from the creation of each database up to January 2011 included Medline, Chinese National Knowledge Infrastructure, EMBASE, Cochrane Library, and bibliographies of review and original articles. Through a systematic literature search for publications, the data from 22 studies were summarized based on their discussions on the result of the CYFRA 21-1 assay in pleural effusion and differential diagnosis evaluation in the Chinese population.

Results: A total of 22 studies were available for analysis, and the high CYFRA 21-1 level in MPE was significantly associated with risk for lung cancer (standardized mean difference $[S M D]=1.65,95 \%$ confidence interval $[\mathrm{CI}]=1.48-1.82, Z=18.97$, $p<0.00001$ ) compared with BPE. The CYFRA 21-1 level in pleural effusion (13 studies) was significantly higher than that in serum $(S M D=1.10,95 \% \mathrm{CI}=0.71-1.48$, $Z=5.59, p<0.00001)$. The risk for squamous cell carcinoma (SCC) for CYFRA 21-1 was $1.03(95 \% \mathrm{Cl}=0.64-1.42, Z=5.15, p<0.00001)$ compared with that of adenocarcinoma ( 8 studies). The sensitivity of CYFRA 21-1 reported in the articles ranged from $46 \%$ to $94 \%$, and the specificity ranged from $57 \%$ to $100 \%$. The summary measure of the test characteristics derived from the summary receiver operating characteristic curve was $81 \%$ for both sensitivity and specificity (17 studies).

Conclusions: The measurement of pleural CYFRA 21-1 is likely to be a useful diagnostic tool for the confirmation of MPE.

Key words: meta-analysis, CYFRA 21-1, pleural effusion, lung cancer, differential diagnosis, tumor marker.

\section{Introduction}

At the beginning of the $21^{\text {st }}$ century, lung cancer has emerged as one of the world's leading causes of mortality [1]. Although this malignancy has been extensively investigated in many fields such as etiology, pathogenesis, and therapeutics, there are still unresolved issues with regard to
Corresponding author: Yang Shuanying PhD Department of Respiratory Medicine Second Affiliated Hospital of Xi'an Jiaotong University 157, Xi 5 Road Xi'an 710004, P.R. China Phone: +86-029-87678001 E-mail: ysy3363930@yahoo.cn, yangshuanying66@163.com 
the diagnosis and therapy of this disease that are intimately correlated with prognosis of patients. Early-stage lung cancer has a better 5-year survival rate compared with cancer diagnosed at a later stage [2]. Delayed diagnosis of lung cancer leads to a high mortality and a dismal treatment outcome. Early diagnosis remains a challenge, and the current research focuses on whether lung cancer-specific molecular alterations can be used as diagnostic signals for the determination of this disease [3]. A common belief is that the earlier the cancer is detected, the better is the probability of reduced mortality and morbidity. The advent of new and emerging molecular, genetic, and imaging technologies has broadened the possible strategies for early detection and prevention, but the beneficial impact of these methods on mortality must be supported by clinical evidence. Molecular markers are being identified as beneficial in enhancing the present ability to predict and detect cancer before the disease progresses as well as detect the earliest signs of impending carcinogenic transformation $[4,5]$. Biological markers are urgently needed to improve the early detection, diagnosis, and treatment of lung tumors. Over the past several years, various promising biomarkers have emerged in the field of lung cancer.

Cytokeratin 19 fragments (CYFRA 21-1) proved to be sensitive and valuable biomarkers for estimation of diagnosis, prognosis, and therapy monitoring of lung cancer [6-11]. The CYFRA 21-1 assay is a test that has been developed for the detection of CYFRA 21-1 in serum, especially in patients with non-small cell lung cancer (NSCLC) [12]. However, reports on pleural effusion in Asian populations are relatively limited. Several studies have investigated the diagnostic evaluation between CYFRA 21-1 level and pleural effusion on cancer epidemiology in Chinese patients. Thus, a medical review of literature was performed, and a meta-analysis was conducted to estimate the magnitude of the association between CYFRA 21-1 level and lung cancer risk in Chinese patients.

\section{Material and methods}

\section{Identification and eligibility of relevant studies}

Published scientific literature in the databases of Medline, Chinese National Knowledge Infrastructure, EMBASE, and Cochrane Library were searched using free text and various medical subject heading terms, such as "pleural effusion", "hydrothorax", "CYFRA 21-1", "tumor marker", "lung cancer”, "cytokeratin 19", and "pulmonary tuberculosis". The search period was from the start of each database up to January 2011, and no language restrictions were added. Moreover, the bibliographical references of the selected articles and the review articles were manually revised. The papers must be case-control or cohort association studies of the CYFRA 21-1 levels in pleural effusion in Chinese patients. The authors of these studies must have provided the sample size, test results $(M \pm S D)$, and information that can help infer the results in the papers. Aside from the database search, papers were also identified by a hand screen from the lists of references of the selected papers and by personally communicating with the authors via e-mail and telephone, if necessary. The search and selection of data were conducted from March 2010 to January 2011.

\section{Data extraction}

To avoid bias and improve reliability of the present research, two investigators extracted data independently and reached a consensus on all items. When the two abstractors did not agree, another expert was invited to discuss the disputed data, and two consistent opinions were adopted. The following data were extracted from each article: first author, date on which the study was published, journal name, the exact data of the total and practical number in case and control groups as well as the test results. The present research was aimed at identifying all trials that reported on the measurement of CYFRA 21-1 levels and investigated benign pleural effusion (BPE) in comparison with malignant pleural effusion (MPE). All studies included in the meta-analysis were unique contributions. If a researcher had published more than one study or if results concerning similar populations were reported several times, then the other studies would be included only if all study populations were independent. All studies underwent an extensive, diagnostic workup to determine the etiology of pleural effusion. The exclusion criteria were as follows: (1) not original research (reviews, editorials, and nonresearch letters), (2) studies not conducted on humans, (3) studies involving patients who had received chemotherapy, and (4) studies that did not use a control group. The methodological quality of the studies was assessed using the guidelines published by the standards for reporting of diagnostic accuracy (STARD, maximum score 25) initiative [13], which set guidelines that aim to improve the quality of reporting in diagnostic studies, and the quality assessment for studies of diagnostic accuracy (QUADAS, maximum score 14) tool [14], in which appraisal is performed by empirical evidence, expert opinion, and formal consensus on assessing the quality of primary studies of diagnostic accuracy. Two reviewers also assessed independently the methodological quality of the studies. The outcome variables were extracted from the BPE and MPE groups. When several measures were reported in the same study, the one with the acknowledged measurement was selected. If the study provided stratum information, the data from all strata were 
collated to utilize the data fully. Studies were included in the meta-analysis if they provided both the sensitivity and specificity of pleural CYFRA 21-1 levels for the diagnosis, or when CYFRA 21-1 values were provided in a scatter plot form. This step facilitated the extraction of test results for individual study subjects. From the selected reports, data were abstracted in duplicate using a standardized form.

\section{Statistical analysis}

For the CYFRA 21-1 levels, mean and standard deviation (SD) were extracted or derived using the data reported. To analyze the differences between BPE and MPE, two different approaches were used, namely, fixed-effect and random-effect models. A fixed-effect model was first performed, assuming a uniform homogeneity of the effect size across all studies. This step was followed by a random-effect model under the assumption of heterogeneity between studies. Both within- and between-study variabilities were considered in the random-effect model, and the effect size was assumed to be drawn from a distribution of the standardized mean difference (SMD). Finally, SMD and their $95 \%$ confidence intervals (Cls) were presented. The $\chi^{2}$ and Fisher's exact tests were used to detect statistically significant heterogeneity. Heterogeneity was also quantified with the $I^{2}$ statistic, which describes the proportion of the total variation in study estimates caused by heterogeneity. Sensitivity was analyzed by omitting each study from the estimated pool at every step. Finally, publication bias was evaluated using funnel plots [15]. Statistical analyses were conducted using Review Manager (version 5, The Cochrane Collaboration). Standard methods recommended for meta-analyses of diagnostic test evaluations were used. Analyses were performed using Stata version 10.0 (Stata Corporation, College Station, TX, USA) and Meta-DiSc (Meta-DiSc for

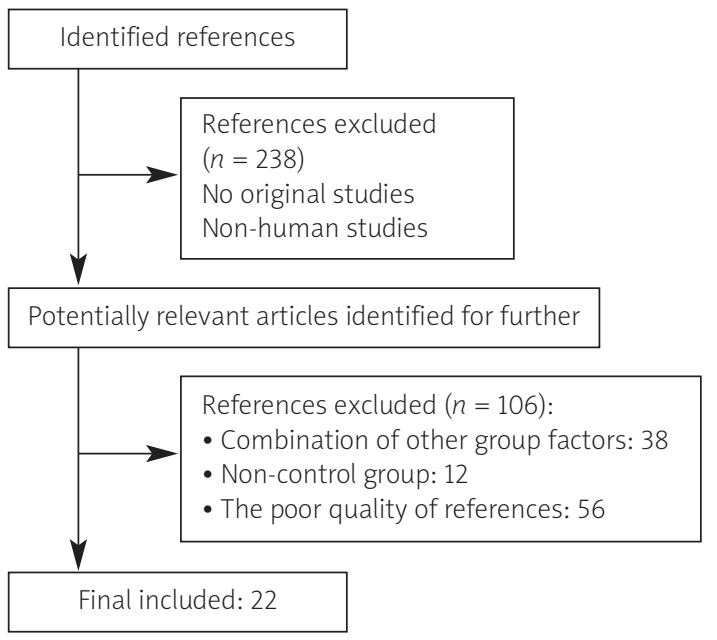

Figure 1. Study selection algorithm
Windows, XI Cochrane Colloquium, Barcelona, Spain, 2003). The following measures of test accuracy were computed for each study: sensitivity, specificity, positive likelihood ratio (PLR), negative likelihood ratio (NLR), and diagnostic odds ratio (DOR). Analysis was based on a summary receiver operating characteristic (SROC) curve. The sensitivity and specificity for the single test threshold identified for each study were used to plot an SROC curve. A random-effect model was used to calculate the average sensitivity, specificity, and other measures across studies. All tests were two-sided, and the significant level was set at 0.05.

\section{Results}

\section{Description of the studies}

A meta-analysis database was established according to the extracted information from each selected paper. Table I lists all essential information such as the publication year, first author, the number of cases (lung cancers), and measure outcomes. Initially, 367 studies were identified, and 128 were case-control studies, in which 22 studies fulfilled the inclusion criteria (Figure 1). The sample sizes varied between 60 [16] and 289 [36] patients, and the subjects fulfilled a wide range of inclusion criteria: adolescents [28], adults, and the elderly [19-30]. The eligible studies included a total of 1653 MPE cases and 1011 BPE controls. The diagnostic criteria defined in each study were consistent. Studies on the histopathology of the pleural biopsy, pleural biopsy specimens, and pathological diagnosis were used as gold standards in all reports. The BPE was involved in non-cancerous effusion, which was induced by other factors such as tuberculosis, heart failure, renal failure, and rheumatic diseases. There were 5 case-control studies that focused on CYFRA 21-1 level as determined by ELISA, whereas seven and ten case-control studies used immunoradiometric assay (IRMA) and electrochemiluminescence assay (ECLA), respectively (Table I).

\section{Test of heterogeneity}

A more common method of indicating the extent of heterogeneity is by a statistical test, often described as Cochran's $\chi^{2}$ test or the Q-test. A $p$-value is frequently quoted as an indication of the extent of between-study variability. The heterogeneity for all 22 case-control studies and the test value of $\chi^{2}$ were analyzed. At first, if the heterogeneity of the studies is considered acceptable, the fixed-effect model and weighted mean difference were used. The $\chi^{2}$ value for the heterogeneity of the 22 case-control studies was 161.18 with 21 degrees of freedom $(d f)$ and $p<0.001$. Another index for the heterogeneity test is the $I^{2}$ value. The $I^{2}$ value describes the percentage of variability in point estimates caused by he- 
Table I. Description of the included studies

\begin{tabular}{|c|c|c|c|c|c|c|c|}
\hline \multirow[t]{2}{*}{ Author, year } & \multirow{2}{*}{$\begin{array}{l}\text { Average age [years] } \\
\text { Benign/malignant }\end{array}$} & \multirow{2}{*}{$\begin{array}{c}\text { Gender } \\
\text { F/M }\end{array}$} & \multicolumn{2}{|c|}{ Hydrothorax group } & \multicolumn{2}{|c|}{ Quality score } & \multirow[t]{2}{*}{ Assay } \\
\hline & & & Benign & Malignant & STARD & QUADAS & \\
\hline Yijiang, 1999 [16] & $33 / 53$ & $28 / 32$ & 30 & 30 & 11 & 9 & ELISA \\
\hline Xiaofu, 1999 [19] & - & $46 / 62$ & 40 & 68 & 5 & 5 & IRMA \\
\hline Huoqiang, 2002 [17] & - & - & 123 & 156 & 5 & 7 & IRMA \\
\hline Aizhen, 2003 [31] & - & - & 38 & 62 & 6 & 7 & IRMA \\
\hline Jianguo, 2003 [32] & $36.4 / 68.7$ & $46 / 130$ & 69 & 107 & 16 & 12 & ECLA \\
\hline Wenfu, 2004 [33] & $46 / 54$ & $38 / 44$ & 42 & 40 & 8 & 6 & IRMA \\
\hline Yalin, 2006 [20] & - & $30 / 32$ & 30 & 32 & 9 & 8 & IRMA \\
\hline Yuseng, 2006 [21] & $59.2 / 60.5$ & $36 / 58$ & 31 & 63 & 11 & 8 & ECLA \\
\hline Zikun, 2008 [22] & $42 / 60$ & $95 / 155$ & 80 & 170 & 16 & 11 & ELISA \\
\hline Lianzhou, 2008 [34] & $32 / 57$ & $17 / 31$ & 18 & 30 & 9 & 6 & ECLA \\
\hline Jiahe, 2008 [23] & $49.4 / 63.7$ & $21 / 35$ & 20 & 36 & 11 & 8 & ECLA \\
\hline Baoxiang, 2008 [24] & $60 / 63$ & $39 / 50$ & 35 & 54 & 16 & 9 & IRMA \\
\hline Xilin, 2008 [25] & $39 / 65$ & $28 / 48$ & 34 & 42 & 10 & 8 & ELISA \\
\hline Guochen, 2008 [26] & 68.5 & $21 / 43$ & 30 & 34 & 15 & 11 & IRMA \\
\hline Fang, 2008 [27] & $60 / 62$ & $45 / 78$ & 45 & 78 & 11 & 9 & ECLA \\
\hline Jin, 2009 [18] & $43.9 / 54.6$ & $48 / 59$ & 48 & 59 & 17 & 14 & ELISA \\
\hline Jing, 2009 [28] & - & $24 / 56$ & 40 & 40 & 11 & 8 & ECLA \\
\hline Yang, 2010 [29] & - & $24 / 69$ & 28 & 65 & 7 & 9 & ECLA \\
\hline Hua, 2010 [30] & $58 / 61$ & $59 / 79$ & 52 & 86 & 6 & 10 & ECLA \\
\hline Hongmei, 2010 [35] & $57.5 / 59.5$ & $48 / 82$ & 50 & 80 & 15 & 12 & ELISA \\
\hline Tian, 2010 [36] & 54.6 & $116 / 212$ & 36 & 253 & 13 & 10 & ECLA \\
\hline Feng, 2010 [37] & $52.1 / 59.3$ & $59 / 101$ & 84 & 76 & 10 & 9 & ECLA \\
\hline
\end{tabular}

F-female, M - male, STARD - standards for reporting diagnostic accuracy (maximum score 25), QUADAS - quality assessment for studies of diagnostic accuracy (maximum score 14), IRMA - immunoradimetric assay, ELISA - enzyme-linked immunosorbent assay, ECLA - electrochemiluminescence assay

terogeneity rather than sampling error. This value may be readily calculated from most published meta-analyses, and a closed form uncertainty interval is available. If the $I^{2}$ value is lower than $50 \%$, the heterogeneity of the studies is considered acceptable. The $I^{2}$ values were $78 \%$ in the first-time analysis in the present study, and both $\chi^{2}$ and $l^{2}$ suggest the existence of heterogeneity of the studies. All studies were carefully reviewed, and the heterogeneity was considered to be derived basically from different assay methods (IRMA, ELISA, and ECLA) and measurement units. All included studies exhibited good clinical homogeneity, considering that clinical homogeneity is critical to the meta-analysis of clinical research. Finally, to reach a credible conclusion, the random-effect model and SMD were selected to perform the meta-analysis. The randomeffect model assumes that the studies included in the meta-analysis are a random sample of a hypothetical population and demonstrate within- and between-study variabilities.

\section{CYFRA $21-1$ in BPE and MPE}

All 22 studies evaluated the CYFRA 21-1 level in MPE compared with BPE. Overall, the eligible studies included 1653 MPE cases and 1011 BPE controls. Five case-control studies determined the CYFRA 21-1 level using ELISA, compared with seven and ten case-control studies that used IRMA and ECLA, respectively. The random-effect model and SMD were used to perform the meta-analysis. The results indicate that the weight of included studies ranged from $3.6 \%$ to $5.9 \%$. The weight derived from SD can be used to evaluate the quality of references. The SMD of the CYFRA 21-1 level in MPE compared with BPE was $1.65(95 \% \mathrm{Cl}=1.48-1.82$; test for heterogeneity $=67.19, p<0.00001, I^{2}=69 \%$; test for overall effect: $Z=18.97, p<0.00001$ ) (Figure 2). There was a significant difference in the CYFRA 21-1 level between the MPE and the BPE. The CYFRA 21-1 level was significantly higher in MPE than that in BPE. In the following analysis of sensitivity, the individual exclusion of studies did not substantially 
Figure 2. Meta-analysis using a random-effect model for the SMD of the CYFRA 21-1 level in BPE and MPE

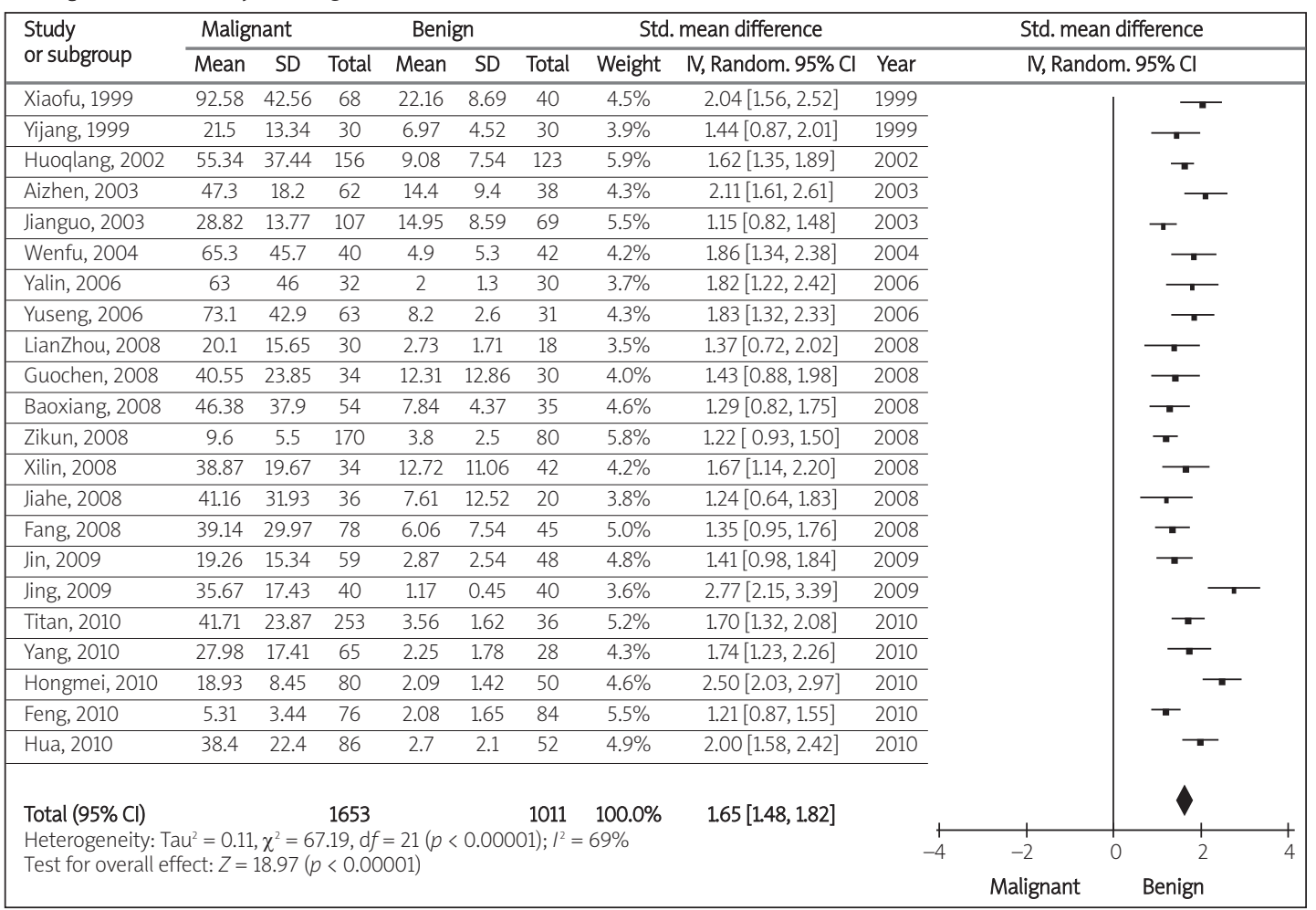

modify the estimators, with SMD values varying between 1.15 and 2.77 .

\section{CYFRA 21-1 in pleural effusion and serum in lung cancer patients}

Thirteen studies compared the CYFRA 21-1 levels between pleural effusion and serum in lung cancer patients, and the eligible studies included 717 cases. Similarly, the random-effect model and SMD were also employed to perform the meta-analysis. The results show that the weight of included studies ranged from $7.4 \%$ to $8 \%$, indicating better consistency. The SMD of the CYFRA 21-1 level in pleural effusion compared with that in the serum of lung cancer patients was $1.10(95 \% \mathrm{Cl}=0.71-1.48$; test for heterogeneity $=134.19, p<0.00001, I^{2}=91 \%$; test for overall effect: $Z=5.59, p<0.00001$ ) (Figure 3). There was a significant difference between the CYFRA 21-1 level in pleural effusion and that in the serum. The CYFRA 21-1 level in the pleural effusion was significantly higher than that in the serum. In the analysis of sensitivity, the individual exclusion of studies did not substantially modify the estimators, with SMD values varying between -0.64 and 2.62 .

\section{CYFRA 21-1 in pleural effusion between squamous cell lung cancer and adenocarcinoma}

Eight studies evaluated the CYFRA 21-1 level in the pleural effusion of SCC and adenocarcinoma patients. Overall, the eligible studies included 268 SCC cases and 320 controls (adenocarcinoma patients). Five case-control studies determined the CYFRA 21-1 level using ECLA, whereas three casecontrol studies used IRMA. The random-effect model and SMD were used to perform the meta-analysis. The results indicate that the weight of included studies ranged from $9.1 \%$ to $16.2 \%$. The SMD of the CYFRA 21-1 level in the pleural effusion of SCC patients compared with adenocarcinoma patients was $1.03(95 \% \mathrm{Cl}=0.64-1.42$; test for heterogeneity $=29.50, p=0.0001, I^{2}=76 \%$; test for overall effect: $Z=5.15, p<0.00001$ ) (Figure 4). There was a significant difference between the CYFRA 21-1 levels in the pleural effusion of SCC and adenocarcinoma patients. The CYFRA 21-1 level in the pleural effusion was higher in SCC patients than that in adenocarcinoma patients. In the analysis of sensitivity, the exclusion of studies individually did not substantially modify the estimators, with SMD values varying between 0.31 and 2.37 .

\section{Publication bias}

To ensure comparability, method comparison and bias evaluation must be performed when the same test is determined by different detecting systems. The outcomes should include the uncertainty to provide reliable data for clinical diagnosis and treatment. The shape of the funnel in the funnel plot analysis of publication biases appeared to be 
Figure 3. Meta-analysis using a random-effect model for the SMD of the CYFRA 21-1 level in pleural effusion and serum in lung cancer patients

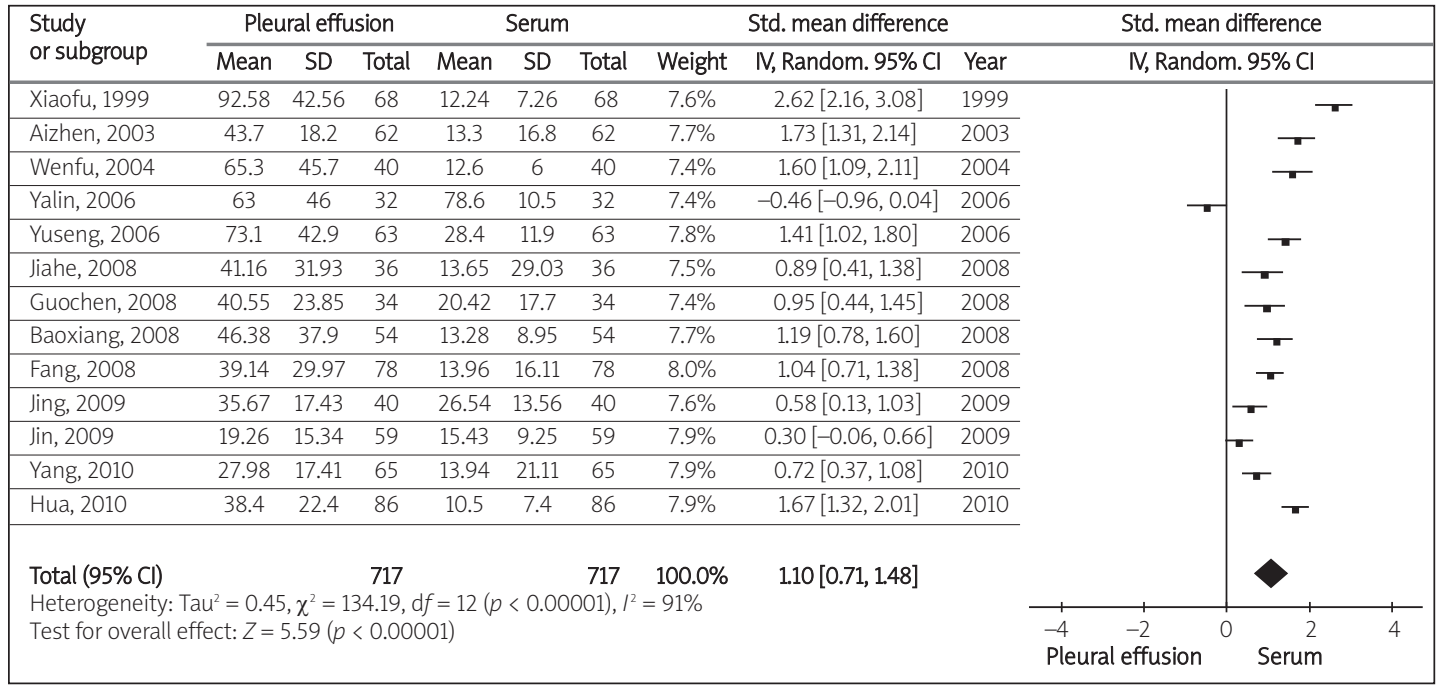

Figure 4. Meta-analysis using a random-effect model for the SMD of the CYFRA 21-1 level in pleural effusion between SCC and adenocarcinoma patients

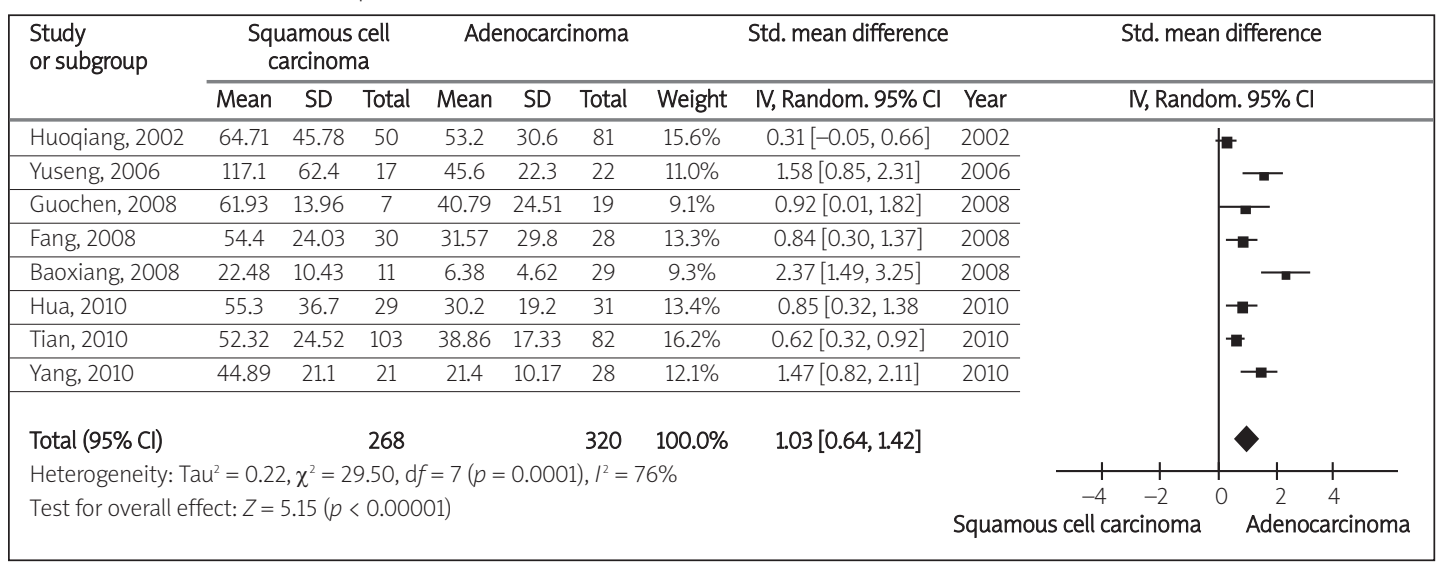

approximately symmetrical (Figure 5 A-C). However, there was some uncertainty because the symmetrical degrees were not satisfied. Egger's test is based on a linear regression of the standard normal deviation against its precision. In the present analysis, the inverse of the standard error is considered as the independent variable and the standardized estimate of the size effect as the dependent variable. The test results were as follows: $t=2.25, \mathrm{~d} f=21$, $p=0.063$, for the CYFRA 21-1 level in MPE compared with BPE; $t=2.30, \mathrm{~d} f=11, p=0.075$, for the CYFRA 21-1 level between pleural effusion and serum in lung cancer patients; and $t=1.107, \mathrm{~d} f=7, p=0.305$, for the CYFRA 21-1 level in pleural effusion in SCC patients compared with adenocarcinoma patients. Begg's test was also performed for the publication bias, and the results of Begg's test indicated the following: SD score was 35.46, and $p=0.185$ for MPE compared with BPE. The fail-safe number signifies the number of negative results necessary to invert the positive effect of studies, which had sta- tistical significance in the meta-analysis. The bigger the fail-safe number is, the more studies with a negative result are needed, indicating the stability of the results of the meta-analysis. The fail-safe number was 4466, 914, and 121 for MPE versus BPE, pleural effusion versus serum, and SCC versus adenocarcinoma meta-analyses, respectively, at a 0.01 level (Table II). These results are consistent with the standard of greater than five times the includedarticle number. Therefore, the Egger and Begg tests, as well as the fail-safe numbers, suggest that publication biases may not have a significant influence on the results.

\section{Study characteristics of CYFRA 21-1 for differential diagnosis}

Seventeen studies evaluated the CYFRA 21-1 level for the utility of this marker in the differential diagnosis of MPE and BPE. The eligible studies included a total of 1771 MPE and BPE cases. Six case-control studies determined the CYFRA 21-1 level using ECLA, 

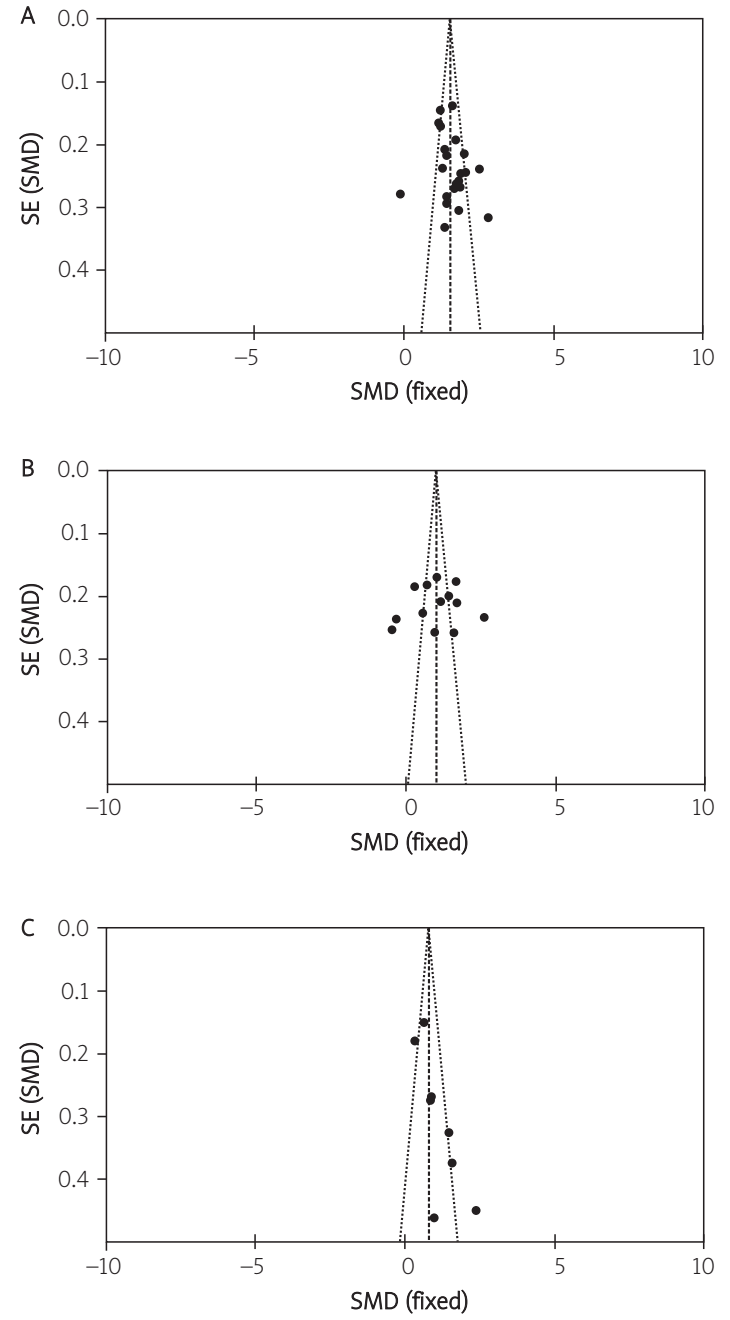

Figure 5. Funnel plot of each meta-analysis performed. The CYFRA 21-1 level in BPE and MPE (A), in pleural effusion and serum in lung cancer patients (B), and in pleural effusion between SCC and adenocarcinoma patients $(\mathrm{C})$

whereas six and five other studies used IRMA and ELISA, respectively. However, the cutoff value was not provided by three studies [22, 28, 31]. The highest sensitivity and specificity were $94 \%$ and $100 \%$, respectively, whereas the lowest were $49 \%$ and $57 \%$, respectively. The number of true positives, false negatives, true negatives, and false positives were extracted from each eligible study to perform the meta-analyses of the diagnostic test (Table III).

\section{Diagnostic accuracy}

The forest plot of the sensitivity and specificity of 17 pleural CYFRA 21-1 assays in the differential diagnosis of MPE and BPE are shown in Figure 6. The average sample size of the included studies was 104 (range: 48-250). The sensitivity ranged from 0.49 to 0.92 (mean: $0.67 ; 95 \% \mathrm{Cl}: 0.64-0.70$ ), whereas the specificity ranged from 0.57 to 1.00 (mean: 0.85;
Table II. The fail-safe number (NFs) of the three metaanalyses

\begin{tabular}{|lccc|}
\hline Meta-analysis & $\boldsymbol{\Sigma} \boldsymbol{Z}$ & NFs 0.05 & NFs 0.01 \\
\hline MPE vs. BPE & 156.1 & 9037 & 4466 \\
\hline Pleural effusion vs. serum & 70.95 & 1858 & 914 \\
\hline $\begin{array}{l}\text { Squamous cell carcinoma } \\
\text { vs. adenocarcinoma }\end{array}$ & 27.93 & 268 & 121 \\
\hline
\end{tabular}

MPE - malignant pleural effusion, BPE - benign pleural effusion

95\% Cl: 0.82-0.87). The PLR was 4.37 (95\% Cl: 3.425.58), the NLR was 0.35 (95\% Cl: $0.28-0.43)$, and the DOR was 15.4 (95\% Cl: 10.54-22.49). The $\chi^{2}$ values of sensitivity, specificity, PLR, NLR, and DOR were $102.06,43.19,28.92,67.90$, and 28.82 , respectively, with all $p$-values less than 0.05 , indicating a significant heterogeneity among studies. An SROC curve plot representing the true-positive against the false-positive rates of individual studies was derived (Figure 7). Most studies had specificity estimates close to 1.00. The specificity estimates of 17 studies were 0.85 . Only one study [33] showed a low specificity of CYFRA 21-1 in the differential diagnosis of pleural effusions $(<0.60)$. By contrast, the sensitivity estimates were relatively low, and the sensitivity was less than 0.50 in only two studies $[27,37]$. The maximum joint sensitivity and specificity was 0.81 [standard error of the mean (SEM), 0.0167], whereas the area under the curve (AUC) was 0.8786 (SEM, 0.0166), indicating that the level of the overall accuracy was medium to high.

\section{Discussion}

The determination of tumor markers in pleural effusions has been proposed as an alternative, noninvasive way of diagnosing pleural malignancy. However, the use of these measurements in clinical practice remains controversial. The CYFRA 21-1 assay, which detects a soluble fragment of cytokeratin 19 that is expressed by all histological types of lung cancer, is capable of detecting epithelial carcinomas, especially SCC. CYFRA 21-1 has been extensively evaluated in the setting of $\operatorname{NSCLC}[38,39]$. Studies on the diagnostic significance of the CYFRA 21-1 level in pleural effusions in differentiating malignant from benign lung disease found CYFRA 21-1 to be a reliable marker. High CYFRA 21-1 levels in the effusion may also be caused by impaired lymphatic drainage or pleural seeding of the tumor. Given that $50 \%$ of MPE is caused by lung cancer and that most advanced-stage patients of lung cancer will have this condition, some experts suggest that CYFRA 21-1 may be a promising tumor marker in this field. This hypothesis is also supported by several studies [8, 12, 40, 41]. However, CYFRA 21-1 also presents various performances in different studies. Porcel et al. 
Table III. Summary data from the 17 studies of the utility of CYFRA 21-1 in the differential diagnosis of MPE and BPE

\begin{tabular}{|c|c|c|c|c|c|}
\hline Study & Number of patients & Assay method & Cut-off & Sensitivity [\%] & Specificity [\%] \\
\hline Yijiang, 1999 [16] & 60 & ELISA & $14.23 \mathrm{ng} / \mathrm{l}$ & 87 & 77 \\
\hline Xiaofu, 1999 [19] & 108 & IRMA & $48.23 \mathrm{ng} / \mathrm{l}$ & 71 & 83 \\
\hline Aizhen, 2003 [31] & 100 & IRMA & Unknown & 94 & 85 \\
\hline Jianguo, 2003 [38] & 176 & ECLA & $20 \mu g / l$ & 63 & 86 \\
\hline Wenfu, 2004 [33] & 82 & IRMA & $15.5 \mu g / l$ & 93 & 57 \\
\hline Yalin, 2006 [20] & 62 & IRMA & $15 \mu \mathrm{g} / \mathrm{l}$ & 60 & 100 \\
\hline Zikun, 2008 [22] & 250 & ELISA & Unknown & 61 & 86 \\
\hline Lianzhou, 2008 [34] & 48 & ECLA & $3.3 \mu \mathrm{g} / \mathrm{l}$ & 80 & 83 \\
\hline Jiahe, 2008 [23] & 56 & ECLA & $20 \mu g / l$ & 65 & 90 \\
\hline Baoxiang, 2008 [24] & 89 & IRMA & $3.3 \mathrm{ng} / \mathrm{ml}$ & 81 & 91 \\
\hline Xilin, 2008 [25] & 76 & ELISA & $34.89 \mathrm{ng} / \mathrm{ml}$ & 68 & 93 \\
\hline Guochen, 2008 [26] & 64 & IRMA & $25 \mathrm{ng} / \mathrm{ml}$ & 79 & 83 \\
\hline Fang, 2008 [27] & 123 & ECLA & $4.74 \mu g / l$ & 49 & 91 \\
\hline Jin, 2009 [18] & 107 & ELISA & $3.3 \mathrm{ng} / \mathrm{ml}$ & 92 & 79 \\
\hline Jing, 2009 [28] & 80 & ECLA & Unknown & 60 & 95 \\
\hline Hongmei, 2010 [35] & 130 & ELISA & $10 \mathrm{ng} / \mathrm{ml}$ & 60 & 78 \\
\hline Feng, 2010 [37] & 160 & ECLA & $3.3 \mathrm{ng} / \mathrm{ml}$ & 46 & 87 \\
\hline
\end{tabular}

[41] reported that in all 416 pleural effusion patients they studied, the sensitivity of CYFRA 21-1 was only $22 \%$, whereas specificity was $100 \%$. Several studies have indicated otherwise $[42,43]$. Considering national, ethnic, and hereditary differences, the evaluation of CYFRA 21-1 in the Chinese population was reviewed in the present study to determine its significance for the Chinese population.

All 22 studies evaluated the CYFRA 21-1 level in MPE compared with BPE. The STARD and QUADAS tools were chosen because they exhibited better validity and reliability evidence in a recently publi-
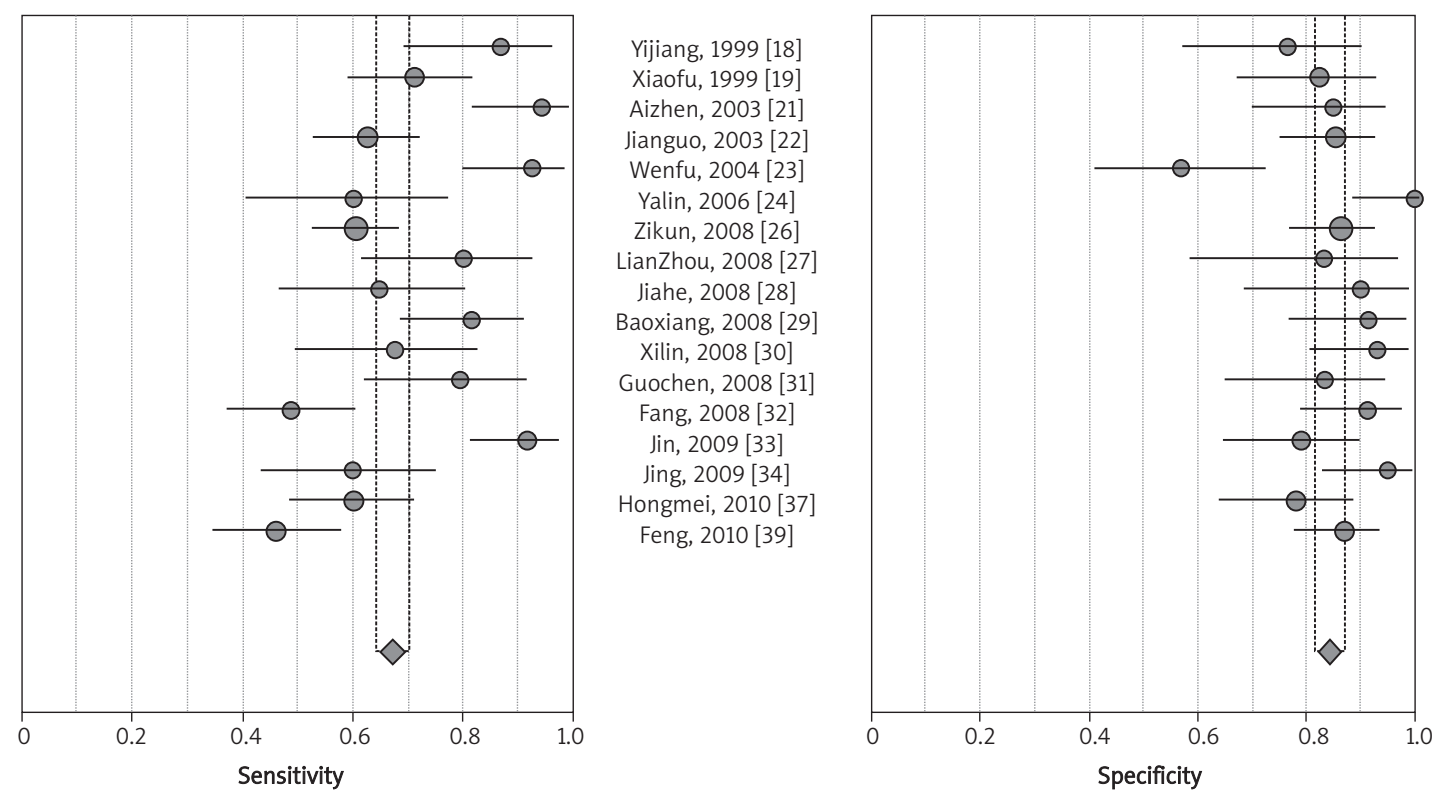

Figure 6. Forest plot of the estimates of sensitivity and specificity for CYFRA 21-1 assays in the differential diagnosis of pleural effusions. The point estimates of sensitivity and specificity from each study are shown as solid circles. Error bars are $95 \% \mathrm{Cls}$ 


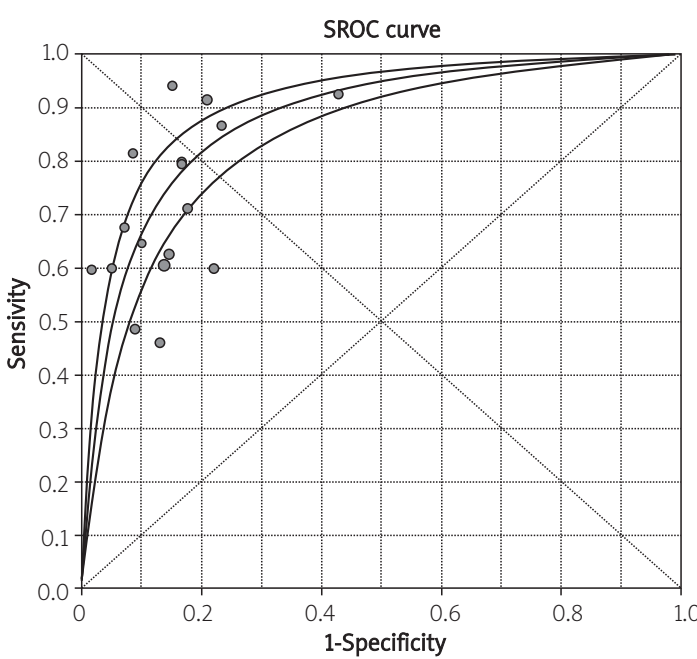

Figure 7. SROC curves of CYFRA 21-1 assays in the differential diagnosis between MPE and BPE. Each solid circle represents each study in the meta-analysis. The size of each study is indicated by the size of the solid circle. The weighted (solid line) regression SROC curves summarize the overall diagnostic accuracy

shed systematic review that evaluated the quality and modifications of these tools [13, 14]. Addressing statistical heterogeneity is one of the most important aspects of many systematic reviews. The interpretative problems depend on how substantial the heterogeneity is because this condition determines the extent to which it might influence the conclusions of the meta-analysis. Therefore, the quantification of the extent of heterogeneity among various studies is essential. All studies were carefully reviewed, and included studies were confirmed to possess good clinical homogeneity. A randomeffect model and SMD were used to combine all studies, although they demonstrated some betweenstudy heterogeneity. In the current meta-analysis, the SMD of the CYFRA 21-1 level in pleural effusion (MPE vs. BPE) in Chinese patients was 1.65 (95\% Cl: 1.48-1.82), which was obtained from 22 studies of 1653 MPE cases and 1011 BPE controls. The results imply that CYFRA 21-1 is a hazard prognostic factor for MPE. Higher CYFRA 21-1 was found in MPE than in BPE. Therefore, the higher the number of patients with lung cancer included in the increased CYFRA 21-1 assay, the better is the test performance, as the present results show, which are consistent with other studies on the CYFRA 21-1 assay [44-46].

Thirteen studies compared the CYFRA 21-1 level between pleural effusion and serum in lung cancer patients. The SMD in the CYFRA 21-1 levels in pleural effusion and serum in lung cancer patients was 1.10 (95\% Cl: 0.71-1.48), indicating a significant difference. The CYFRA 21-1 level was significantly higher in pleural effusion than that in serum, indicating that more CYFRA 21-1 was secreted to pleural effusion than to serum at the same time. In the pleu- ral effusion assay based on CYFRA 21-1, the reasoning efficiency seemed to be improved compared with serum and can be used in the differential diagnosis of lung cancer. The research involving 343 healthy persons, 474 patients with a benign disease, and 4440 patients with a malignant disease, 2453 of whom suffer from NSCLC, has found that the sensitivity and specificity of CYFRA 21-1 in SCC are higher than other histological types of lung cancer [47]. A previous study has also reported the positive rate of CYFRA 21-1 in SCLC compared with other type of lung cancers [48]. In the current meta-analysis, the results show that CYFRA 21-1 had a higher significance in SCC than in adenocarcinoma patients and indicated that this marker can improve the sensitivity, as well as accuracy, in the detection of SCC. Common findings suggest that pleural effusion concentration of CYFRA 21-1 is suitable for use in the early diagnosis of SCC [49]. Several studies have found that the CYFRA 21-1 assay is a better tumor marker of MPE [50,51]. The sensitivity of the CYFRA 21-1 assay most likely depends on the prevalence of squamous cell malignancies in the studied population. More studies on the diagnostic significance of the CYFRA 21-1 level in pleural effusions in differentiating malignant from benign lung disease $[52,53]$ have found that CYFRA $21-1$ is a reliable marker. The current research is consistent with those findings.

Choosing an outcome measurement method is a crucial step in the evaluation of CYFRA 21-1 in differentiating MPE from BPE. In clinical practice, different measurement methods for the diagnosis of lung cancer are always employed to detect the CYFRA 21-1 level. The present meta-analysis demonstrates that the concentration of pleural CYFRA 21-1 has high specificity $(0.85 ; 95 \% \mathrm{Cl}: 0.82-0.87)$ and PLR (4.37; $95 \% \mathrm{Cl}: 3.42-5.58)$ for the differential diagnosis of pleural effusions. The summary estimate of sensitivity, however, was only 0.67 ( $95 \% \mathrm{Cl}$ : 0.64-0.70). A PLR of 4.37 suggests that patients with MPE have a nearly fivefold higher chance of being positive for CYFRA 21-1 compared with patients with BPE. This ratio suggests a potential role of CYFRA 21-1 determination in discerning the properties of pleural effusion. However, these tests maximize specificity at the cost of sensitivity, and this trade-off has significant clinical implications. CYFRA 21-1 has a relatively low sensitivity and NLR values that do not exclude BPE when a patient's pleural CYFRA 21-1 concentration is lower than the cutoff value. A negative test, therefore, does not signify the absence of malignancy, and patients with negative CYFRA 21-1 results have a chance of having malignancy. The DOR is an indicator of test accuracy [54] that combines the data of sensitivity and specificity into a single number. Generally speaking, the DOR values range from 0 to infinity, with higher DOR values showing higher accuracy. In the current meta-analysis, the 
mean DOR was 15.4, indicating that the CYFRA 21-1 assay could be advantageous in the diagnosis of MPE. The definitive diagnosis of pleural effusion usually requires pleural biopsies of adequate size for histopathological analysis, ideally obtained by pleuroscopy. When malignancy is suspected on pleural cytology and/or pleural needle biopsy, a test with a good NLR for MPE would avoid diagnostic pleuroscopy. The present meta-analysis shows that the pleural CYFRA 21-1 assay is useful in the differential diagnosis of MPE and BPE. The SROC curve has been recommended to represent the performance of a diagnostic test, and the area under the SROC curve (AUC) is not only useful to summarize the curve but is also quite robust to heterogeneity $[54,55]$. A previous study [56] has shown that to demonstrate excellent accuracy, the AUC should be in the region of 0.97 or above. An AUC of 0.93 to 0.96 is very good, whereas 0.75 to 0.92 is good. An AUC less than 0.75 can still be reasonable, but the test has obvious deficiencies in its diagnostic accuracy. In the current analysis, the AUC of CYFRA 21-1 was 0.87 , which varied between 0.75 and 0.92 . This result shows that the pleural fluid CYFRA 21-1 has good accuracy in terms of the differential diagnosis of pleural effusion. Based on the present data, the authors believe that every patient with an unexplained pleural effusion should undergo thoracocentesis with CYFRA 21-1 evaluations. Patients with negative cytological examinations and positive CYFRA 21-1 levels should undergo further invasive procedures, and the final step should depend on the demonstration of positive cytology or biopsy of the pleura, resulting in management decisions.

\section{Acknowledgments}

This work was supported by Fundamental Research Funds for the Central Universities in China.

\section{References}

1. Little AG, Gay EG, Gaspar LE, Stewart AK. National survey of non-small cell lung cancer in the United States: epidemiology, pathology and patterns of care. Lung Cancer 2007; 57: 253-60.

2. Alberg AJ, Ford JG, Samet JM. Epidemiology of lung cancer: ACCP evidence-based clinical practice guidelines $\left(2^{\text {nd }}\right.$ edition). Chest 2007; 132 (3 Suppl): 29S-55S.

3. Ocak S, Sos ML, Thomas RK, Massion PP. High-throughput molecular analysis in lung cancer: insights into biology and potential clinical applications. Eur Respir J 2009; 34: 489-506.

4. Dunn BK, Wagner PD, Anderson D, Greenwald P. Molecular markers for early detection. Semin Oncol 2010; 37: 224-42.

5. El Bassiouny A, Abou-Shousha T, Moussa M, et al. Hepatic mRNA expression of histone (H3): an early predictor of tumorgenic changes in chronic hepatitis C. Arch Med Sci 2009; 5: 506-12.

6. Molina R, Filella X, Augé JM, et al. Tumor markers (CEA, CA 125, CYFRA 21-1, SCC and NSE) in patients with non-small cell lung cancer as an aid in histological diagnosis and prognosis. Comparison with the main clinical and pathological prognostic factors. Tumor Biol 2000; 24: 209-18.
7. Holdenrieder S, Stieber P, Von Pawel J, et al. Early and specific prediction of the therapeutic efficacy in non-small cell lung cancer patients by nucleosomal DNA and cytokeratin19 fragments. Ann N Y Acad Sci 2006; 1075: 244-57.

8. Vollmer RT, Govindan R, Graziano SL, et al. Serum CYFRA 21-1 in advanced stage non-small cell lung cancer: an early measure of response. Clin Cancer Res 2003; 9: 1728-33.

9. Ebert W, Muley T. CYFRA 21-1 in the follow-up of inoperable non-small cell lung cancer patients treated with chemotherapy. Anticancer Res 1999; 19: 2669-72.

10. Ando S, Suzuki M, Yamamoto N, lida T, Kimura H. The prognostic value of both neuron-specific enolase (NSE) and Cyfra21-1 in small cell lung cancer. Anticancer Res 2004; 24: 1941-6.

11. Pujol JL, Molinier O, Ebert W, et al. CYFRA 21-1 is a prognostic determinant in non-small-cell lung cancer: results of a meta-analysis in 2063 patients. Br J Cancer 2004; 90: 2097-105.

12. Rastel D, Ramaioli A, Cornillie F, Thirion B. CYFRA 21-1, a sensitive and specific new tumour marker for squamous cell lung cancer. Report of the first European multicentre evaluation. CYFRA 21-1 Multicentre Study Group. Eur J Cancer 1994; 30A: 601-6.

13. Bossuyt PM, Reitsma JB, Bruns DE, et al. Towards complete and accurate reporting of studies of diagnostic accuracy: the STARD initiative. Standards for Reporting of Diagnostic Accuracy. Clin Chem 2003; 49: 1-6.

14. Whiting P, Rutjes AW, Reitsma JB, Bossuyt PM, Kleijnen J. The development of QUADAS: a tool for the quality assessment of studies of diagnostic accuracy included in systematic reviews. BMC Med Res Methodol 2003; 3: 25.

15. Egger M, Davey Smith G, Schneider M, Minder C. Bias in meta-analysis detected by a simple, graphical test. BMJ 1997; 315: 629-34.

16. Yijang $\mathrm{H}$, Libai $\mathrm{H}$, Chuanwan Z. The value of CYFRA 21-1 in pleural liquid for the differential diagnosis of malignant and tuberculous [Chinease]. J Clin Intern Med 1999; 16: 23-4.

17. Huoqiang W, Jisheng M. The significance of CYFRA 21-1, CEA and NSE in pleural effusion for the diagnosis of lung cancer [Chinease]. Chin J Lab Diagn 2002; 6: 22-4.

18. Jing L, Jing $X$, Lina $W$, et al. CEA, CYFRA 21-1, TSGF joint simultaneous detection for the diagnosis of malignant pleural effusion [Chinese]. Medical Information 2009; 22: 1292-3.

19. Xiaofu Z, Yunxia L, Guangxian W. Study on diagnosis value of analyzing CYFRA 21-1 and CEA in benign and malignant pleural effusion [Chinese]. Chin J Antitubercul 1999; 21: 97-9.

20. Yalin $H$, Wenying Z, Huizhen L. The differential diagnosis is of pleural effusion by multiple methods combination [Chinease]. Shaanxi Med J 2006; 35: 37-9.

21. Yusheng P, Peng W, Jian D. Clinical value of detection of CYFRA21-1, NSE and CA72-4 in pleural effusion and serum in lung cancer patients [Chinese]. Int J Lab Med 2006; 27: 686-7.

22. Zikun L, Shaofa X, Zhidong L, et al. The clinical significance of tumor markers detection in pleural effusion in the patients with lung cancer [Chinese]. China Cancer 2008; 17: 249-51.

23. Jiahe S, Weiqiang X, Qingxia X. Detection of CEA, CYFRA 21-1 and NSE in serum and pleural fluid cancerous pleural effusion patients [Chinese]. Journal of Zhengzhou University (Medical Sciences) 2008; 43: 768-71.

24. Baoxiang B, Nan H, Fenglei W, et al. Clinical diagnostic significance of combined detection of serum and pleural effusion levels of CEA, NSE, CYFRA21-1, SCC-Ag in patients with lung cancer. J Radioimmunol 2008; 21: 391-4.

25. Xilin X, Dong L, Xiaoyuan W, et al. The significance of levels of CYFRA21-1 and VEGF in pleural effusion in differentia 
ting tuberculosis and malignant pleural effusions [Chi nese]. Clinical Misdiagnosis Mistherapy 2008; 21: 9-10.

26. Guocheng W, Huacheng H, Minghua S, et al. Value of combined detection of CEA, CYFRA 21-1 and NSE in differentia diagnosis of pleural effusion in elderly patients. Pract Geriatr 2008; 22: 303-5.

27. Fang H, Xiaoli W, Lian Y, et al. Clinical value of combined determination of serum and pleural effusion level of CEA CYFRA21-1, TPS in the diagnosis of lung cancer [Chinese]. Chin J Cell Mol Immunol 2008; 24: 370-2.

28. Jing Z, Guohui L, Shuzhi X, Jianfeng Z. Value of CEA, NSE, CYFRA21-1 in pleural fluid and serum for the diagnosis of malignant pleural exudates. J Clin Pulm Med 2009; 14: 484-4.

29. Yang J, Xiaoxian L, Bing L. Clinical application of combined detection of tumor markers in pleural effusion [Chinese]. Mil Med J S Chin 2010; 24: 199-200.

30. Hua G, Bingchang Z, Weihua F, et al. Value of the NSE, CYFRA21- 1 and CEA examination in lung cancer diagnosis [Chinese]. Shandong Medical J 2010; 50: 11-2.

31. Aizhen L, Xiaojuan L, Zhenqian L. Clinic value the mensurate of CYFRA 21-1 in patients' serum and thoracic fluid with NSCLC [Chinese]. China Journal of Modern Medicine 2003; 13: 57-8.

32. Jianguo W, Shijun Z, Ruihua L, Ming Q. Combined detection of CEA, CYFRA 21-1, NSE and SF levels in Che st effusion fluid for differentiation of malignant hydrothorax from Tuberculous hydrothorax. J Radioimmunol 2003; 16: 260-1.

33. Wenfu S, Genyun L, Kaisheng Y. Study on diagnostic value of analyzing CYFRA 21-1 combined with CEA in benign and malignant pleural effusions [Chinese]. Hainan Med J 2004; 15: 25-6.

34. Lianzhou C, Lu Z, Wen L, et al. The application of tumor marker detection in pleural effusion for differentiation of benign diseases from malignant ones [Chinese]. China Tropical Medicine 2008; 8: 1709-10.

35. Hongmei L, Junhua F, Yuande X, et al. Diagnostic significance of combining telomerase activity with CYFRA 21-1 level in differentiating malignant pleural effusion caused by lung cancer from benign pleural effusion [Chinese]. Chin J Lung Cancer 2010; 13: 652-4.

36. Tian C, Xianhua L, Wenzhong S. Relationship between the pleural effusion levels of CYFRA 21-1, NSE, CEA and histological types as well as TNM stages of the tumor in patients with lung cancer [Chinese]. J Radioimmunol 2010 23: 46-8.

37. Feng G, Chunlong W, Shiyong C, et al. Clinical value of tumor markers assay in differential diagnosis of pleural effusion [Chinese]. Chinese Journal of Health Laboratory Technology 2010; 20: 1127-8.

38. Lai RS, Chen CC, Lee PC, Lu JY. Evaluation of cytokeratin 19 fragment (CYFRA 21-1) as a tumor marker in malignant pleural effusion. JPN J Clin Oncol 1999; 29: 421-4.

39. Dejsomritrutai W, Senawong S, Promkiamon B. Diagnostic utility of CYFRA 21-1 in malignant pleural effusion. Respirology 2001; 6: 213-6.

40. Paganuzzi M, Onetto M, Marroni P, et al. Diagnostic value of CYFRA 21-1 tumor marker and CEA in pleural effusion due to mesothelioma. Chest 2001; 119: 1138-42.

41. Porcel JM, Vives M, Esquerda A, Salud A, Pérez B, Rodríguez Panadero F. Use of a panel of tumor markers (carcinoembryonic antigen, cancer antigen 125, carbohydrate antigen 15-3, and cytokeratin 19 fragments) in pleural fluid for the differential diagnosis of benign and malignant effusions. Chest 2004; 126: 1757-63.

42. Lee JH, Chang JH. Diagnostic utility of serum and pleural fluid carcinoembryonic antigen, neuron-specific enolase, and cytokeratin 19 fragments in patients with effusions from primary lung cancer. Chest 2005; 128: 2298-2303.

43. Shitrit D, Zingerman B, Shitrit AB, Shlomi D, Kramer MR. Diagnostic value of CYFRA 21-1, CEA, CA 19-9, CA 15-3, and CA 125 assays in pleural effusions: analysis of 116 cases and review of the literature. Oncologist 2005; 10: 501-7.

44. Huang WW, Tsao SM, Lai CL, SU CC, Tseng CE. Diagnostic value of Her-2/neu, Cyfra 21-1, and carcinoembryonic antigen levels in malignant pleural effusions of lung adenocarcinoma. Pathology 2010; 42: 224-8.

45. Korczynski P, Krenke R, Safianowska A, et al. Diagnostic utility of pleural fluid and serum markers in differentiation between malignant and non-malignant pleural effusions. Eur J Med Res 2009; 14 Suppl 4: 128-33.

46. Gu P, Huang G, Chen Y, Zhu C, Yuan J, Sheng S. Diagnostic utility of pleural fluid carcinoembryonic antigen and CYFRA 21-1 in patients with pleural effusion: a systematic review and meta-analysis. J Clin Lab Anal 2007; 21: 398-405.

47. Pavićević R, Bubanović G, Franjević A, Stancić-Rokotov D, Samarzija M. CYFRA 21-1 in non-small cell lung cancer-standardisation and application during diagnosis. Coll Antropol 2008; 32: 485-98.

48. Matsuoka K, Sumitomo S, Nakashima N, Nakajima D, Misaki N. Prognostic value of carcinoembryonic antigen and CYFRA21-1 in patients with pathological stage I non-smal cell lung cancer. Eur J Cardiothorac Surg 2007; 32: 435-9.

49. Alataș F, Alataș O, Metintaș M, Colak O, Harmanci E, Demir S. Diagnostic value of CEA, CA 15-3, CA 19-9, CYFRA 21-1, NSE and TSA assay in pleural effusions. Lung Cancer 2001; 31: 9-16.

50. Kuralay F, Tokgöz Z, Cömlekci A. Diagnostic usefulness of tumour marker levels in pleural effusions of malignant and benign origin. Clin Chim Acta 2000; 300: 43-55.

51. Satoh H, Sumi M, Yagyu H, et al. Clinical evaluation of CYFRA 21-1 in malignant pleural fluids. Oncology 1995; 52: 211-4.

52. Toumbis M, Rasidakis A, Passalidou E, et al. Evaluation of CYFRA 21-1 in malignant and benign pleural effusions. Anticancer Res 1996; 16: 2101-4.

53. Glas AS, Lijmer JG, Prins MH, Bonsel GJ, Bossuyt PM. The diagnostic odds ratio: a single indicator of test performance. J Clin Epidemiol 2003; 56: 1129-35.

54. Lijmer JG, Bossuyt PM, Heisterkamp SH. Exploring sources of heterogeneity in systematic reviews of diagnostic tests. Stat Med 2002; 21: 1525-37.

55. Walter SD. Properties of the summary receiver operating characteristic (SROC) curve for diagnostic test data. Stat Med 2002; 21: 1237-56.

56. Jones CM, Athanasiou T. Summary receiver operating characteristic curve analysis techniques in the evaluation of diagnostic tests. Ann Thorac Surg 2005; 79: 16-20. 